\title{
Collective Planar Actuation of Miniature Magnetic Robots Towards Individual Robot Operation
}

\author{
Jiří Kuthan, Martin Juřík, Martin Vítek, and František Mach
}

\begin{abstract}
Magnetic actuation technique for the collective operation of untethered miniature robots by uniform control input is presented. The technique allows us to actuate multiple robots simultaneously and enables us to prohibit the actuation of selected ones while others are still operated. Non-actuation of selected robots is achieved in the area, where the global magnetic field generated by coplanar coils is locally disrupted. The position of the area in the space can be changed with a discrete step and it allows us to operate multiple robots at the same time dynamically. The proposed technique is studied by numerical simulations and experimental verification on a laboratory prototype of the system.
\end{abstract}

\section{INTRODUCTION AND MOTIVATION}

Untethered miniature robots operated by external magnetic field seems to be an up-and-coming technique for many future technologies. Most of the promising application was developed in the field of medicine, such as drug delivery [1] and bio-object manipulation [2] [3], or additive manufacturing techniques for functional materials [4] [6] [5]. The collective operation of miniature robots and its swarms remains one of the significant challenges that originate by its nature from physics when space is scaled down [7] [8]. As an example, consider the system discussed below, which allows operating robots with load capacity more than 40 times its weight, but it is still only a few grams of effectively manipulated mass. Collective actuation will then increase the capabilities of untethered magnetic robots at small scales. [9]

The paper discusses magnetically guided actuation of multiple identical miniature robots towards their collective operation on planar surfaces. The proposed technique is based on coplanar coils topology described in Sec. II and take advantage of the idea to prevent the actuation of selected robots while others can continue in operation. [10] [11] [12] The main advantage is the possibility of dynamic formation of the area where robot actuation is prohibited. Attention is paid to the numerical simulation describing the presented technique (Sec. III-A) and the experimental verification (Sec. IV) on the laboratory prototype Gryllus ${ }^{2}$ (Sec. II-B).

\section{System ConcePt AND DESIGN}

The system under discussion allows the actuation of miniature untethered robots on planar surfaces. The motion of the robots originates in the magnetic field generated by the

This research has been supported by the Ministry of Education, Youth and Sports of the Czech Republic under the RICE - New Technologies and Concepts for Smart Industrial Systems, project No. LO1607 and by the University of West Bohemia under the project SGS-2018-043.

Authors are with Faculty of Electrical Engineering, University of West Bohemia in Pilsen, Czech Republic \{kuthanji, mjurik, martinv, fmach\}@kte.zcu.cz coils system illustrated in Fig. 1. The system takes advantage of the coplanar coils topology (fast dynamic response, low spatial demands and simple scaling), easy to manufacture robot (simple production at mesoscale), and simple operation (high repeatability and precision based on discrete spatially distributed source of the magnetic field). [13]

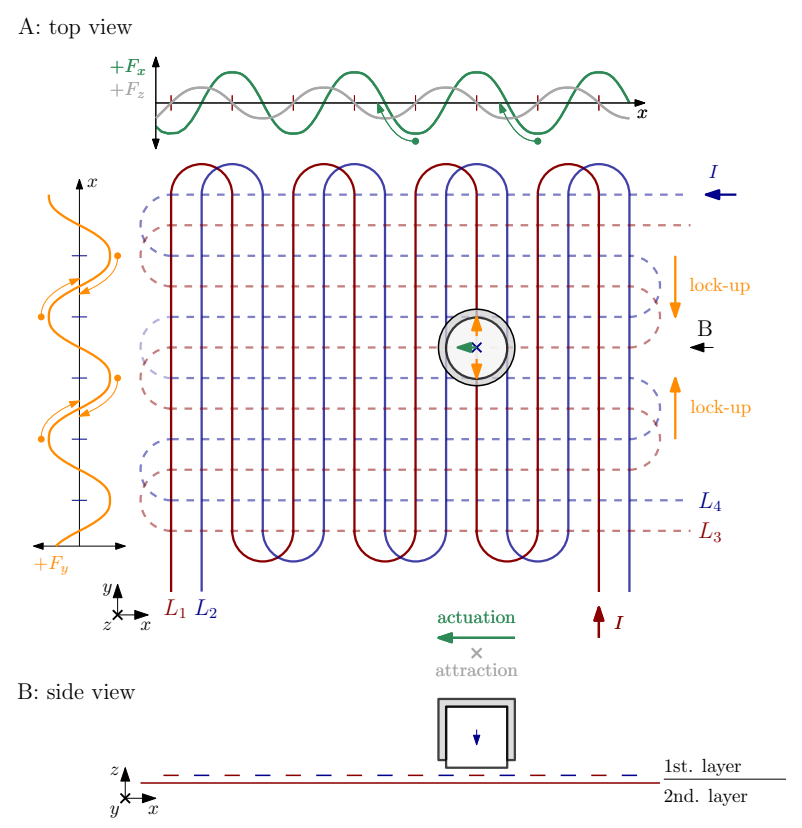

Fig. 1. Illustration of the system topology and robot operation. Two pairs of coplanar coils allow 2-DOF actuation of the robot composed of permanent magnet and non-magnetic body (full lines represent coils $L_{1}, L_{2}$ actuating robot in the $x$-direction and dashed lines coils $L_{3}, L_{4}$ in a different layer actuating robot in the $y$-direction). Forces representing actuation, attraction and also lock-up of the robot are presented.

\section{A. Single robot actuation}

The robot represents a simple cylindrical permanent magnet $(1 \times 0.5 \mathrm{~mm})$ with magnetization along its main axis (robot can be easily joined, see. Sec. II-B). The attractive force $F_{\mathrm{x}, \mathrm{y}}$ and repulsive force $F_{\mathrm{z}}$ acting on the robot is generated by excitation of the coplanar coils $\left(L_{1}, L_{2}\right)$ and is periodic throughout the whole working area $6000 \mathrm{~mm}^{2}$. The continuous movement of the robot is then achieved by sequential excitation of the pair coils for each direction (two pairs of orthogonal coplanar coils are necessary for 2-DOF motion).

The actuation of the robot is subject to uncertainty, which is necessary to eliminate motion over long distances. An 
elimination technique is based on the superposition of the magnetic field generated by both pairs of orthogonal coplanar coils. While one pair of coils generates a time-variable magnetic field (actuation field), the second pair of the coils generate a time-invariant magnetic field (lock-up field) that keeps the robot exactly between the threads of the coil (see Fig. 1). [14]

\section{B. Multiple robot actuation}

While single robot actuation is a simple control issue, an individual actuation of multiple identical robots is a challenge. The periodicity of the actuation field generated by coplanar coils prevents the independent actuation of several identical robots. All robots in the working area are actuated simultaneously, and the system can be considered as underactuated (all robots in the working area move in the same direction and at the same speed with uniform actuation input). [15] [16]

Individual actuation of identical robots can be achieved by spatial separation of coplanar coils to independent segments. Each segment can control a single robot independently or multiple robots simultaneously. The main disadvantage of a segmented design is a large number of switching elements and demand scale reduction. [17] [18] [12]

The reversed approach seems to be a promising way for the collective actuation of identical magnetic robots. While the actuation of selected ones is not possible, the restriction of other robot actuation is feasible. This can be done by the superposition of two magnetic fields - the actuation field and the freezing field. The idea is to locally disturb the magnetic field generated by the additional coils (freezing area). [10] [11]

The additional coplanar coil can be added to achieve the freezing area in a specific part of the working area. The freezing field is then generated by excitation of an additional pair of coplanar coils $\left(L_{3}, L_{4}\right)$ inversely to the primary coils $\left(L_{1}, L_{2}\right)$ of all steps in the control sequence. However, this basic topology does not allow the spatial transition of the freezing area, and its position is then clearly defined by the location of the additional coil. [12]

A more interesting topology seems to be a system of two similar pairs of coplanar coils illustrated in Fig. 2. In this topology, both pairs of coplanar coils slightly differ in distance between the individual wires $\left(d_{L 1}=d_{L 2}\right.$ and $d_{L 3}=$ $\left.d_{L 4}\right)$. Excitation of all coils by a specific sequence allows robots actuation together with the formation of multiple freezing areas through the working area of the system. The most important feature of this topology is the possibility to change the position of the freezing area only by the change of the excitation sequence of coplanar coils (see Sec. III). Therefore, the topology is studied in the paper in more detail based on numerical simulations and experiments.

\section{FREEZING AREA FORMATION}

The proposed topology of coplanar coils (see Fig. 2) allows creating multiple freezing areas while full control of the robots outside is preserved. Actuation of all robots

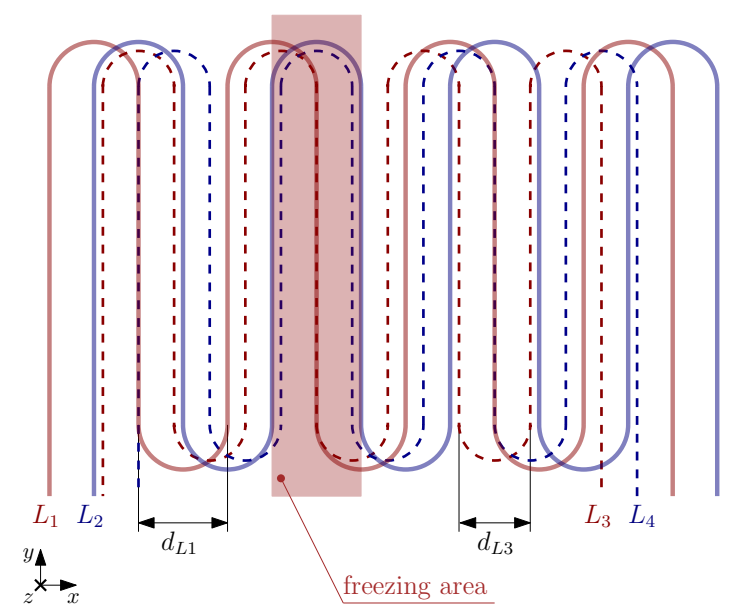

Fig. 2. The conceptual topology of the system for 1-DOF actuation of the robots. The figure shows two coplanar coils with a slightly different thread pitch $d_{L_{1}}$ and $d_{L_{3}}$ which allows to creating a dynamic freezing area.

together is achieved by the excitation of the primary coils $\left(L_{1}, L_{2}\right)$ by the appropriate control sequence described is Sec. II-A. In the case of collective multi-robot actuation, both pairs of coplanar coils in each actuation direction have to be excited (for simplicity, 1-DOF actuation is discussed).

The control sequence ${ }^{1}$ is essential for a understanding of the presented actuation technique. It is necessary to achieve sequential coils excitation (four steps are needed for continual robots movement in each direction) ensuring the robots actuation outside the freezing areas. All possible variants of both coplanar coils excitation were studied by numerical model and possible variants for presented topology were collected in Tab. I. Each control sequence represents the specific position of the freezing areas (see Fig. 3). The actuation of the robot outside the freezing area is then provided by four steps for each sequence. All sequences are collected in Tab. I together with an example of all steps in sequence 3 . The Fig. 3 shows the results of numerical simulation.

\section{A. Numerical simulation}

The numerical simulation was performed to verify the formation of freezing areas while full actuation of the robot outside is achieved and design coplanar coils system for Gryllus $^{2}$ prototype used for hardware experiments (see Sec. IV and Sec. II-B). Formulated mathematical model (see Sec. V-B) was solved numerically in the 3D Cartesian system for the 1D actuation of the robot. For numerical model calibration, magnetic flux density $\boldsymbol{B}$ on the surface of permanent magnets were measured. At a distance of $0.5 \mathrm{~mm}$ has been measured value $|\boldsymbol{B}|=90 \mathrm{mT}-107 \mathrm{mT}$. The remanent magnetization $B_{\mathrm{r}}=1.2 \mathrm{~T}$ was determined based on the results.

From the design point of view, only one pair of coplanar coils can be placed in a single layer. Then, one layer includes

\footnotetext{
${ }^{1}$ Control sequence presents excitation of all coplanar coils $\left(L_{1}, L_{2}, L_{3}\right.$, $L_{4}$ ) needed for robot actuation in one direction.
} 
TABLE I

CONTROL SEQUENCES

\begin{tabular}{|c|c|c|c|c|c|}
\hline \multicolumn{6}{|c|}{ All sequences for freezing area formation } \\
\hline & L1 & $\mathrm{L} 2$ & L3 & $\mathrm{L} 4$ & \\
\hline S1 & $+\mathrm{I}$ & $+\mathrm{I}$ & $+\mathrm{I}$ & $+\mathrm{I}$ & sequence 1 \\
\hline $\mathrm{S} 2$ & $+\mathrm{I}$ & $-\mathrm{I}$ & $+\mathrm{I}$ & $-\mathrm{II}$ & sequence 1 \\
\hline S3 & $+\mathrm{I}$ & $+\mathrm{I}$ & $-\mathrm{I}$ & $-\mathrm{I}$ & sequence 2 \\
\hline S4 & $+\mathrm{I}$ & $-\mathrm{I}$ & $-\mathrm{I}$ & $+\mathrm{I}$ & sequence 2 \\
\hline S5 & $+\mathrm{I}$ & -I & $-\mathrm{I}$ & $-\mathrm{I}$ & sequence 3 \\
\hline S6 & $-\mathrm{I}$ & $-\mathrm{I}$ & $-\mathrm{I}$ & $+\mathrm{I}$ & sequence 3 \\
\hline S7 & $-\mathrm{II}$ & $-\mathrm{I}$ & $+\mathrm{I}$ & $-\mathrm{I}$ & sequence 4 \\
\hline S8 & $-\mathrm{I}$ & $+\mathrm{I}$ & $-\mathrm{I}$ & $-\mathrm{I}$ & sequence 4 \\
\hline \multicolumn{6}{|c|}{ All steps for robot actuation for sequence 3} \\
\hline & L1 & L2 & L3 & L4 & ref to fig. 3 \\
\hline 1 step & $+\mathrm{I}$ & -I & $-\mathrm{I}$ & $-\mathrm{I}$ & $+1 \times S 5$ \\
\hline 2 step & $+\mathrm{I}$ & $+\mathrm{I}$ & $+\mathrm{I}$ & $-\mathrm{II}$ & $-1 \times S 6$ \\
\hline 3 step & $-\mathrm{I}$ & $+\mathrm{I}$ & $+\mathrm{I}$ & $+\mathrm{I}$ & $-1 \times S 5$ \\
\hline 4 step & $-\mathrm{I}$ & -I & -I & $+\mathrm{I}$ & $+1 \times S 6$ \\
\hline
\end{tabular}

two coils that allow the actuation or the freezing of the robot in one direction. The different excitation currents have to be applied to the different layers because of the reduction of the magnetic flux density and then also the force acting on the robot with increasing distance from the actuation surface. The sufficient currents for actuation of the robot in the first and second layer, $0.2 \mathrm{~A}$ and $0.5 \mathrm{~A}$ respectively, has been verified experimentally.

Figure 3 collects the results of the performed simulations. All data were obtained by superposition of the forces generated by individual layers (each layer contains one pair of coplanar coils) and an appropriate control sequence (see Tab. I). The superposition was based on discrete characteristics of the force $F_{\mathrm{x}}$ (actuation force) and $F_{\mathrm{z}}$ (attraction force $)^{2}$ interpolated over the working area because of computational demands. The force superposition has been verified by a full model (model respecting the interaction of individual layers) in the freezing area and outside. The superposition causes only negligible errors to the result.

The results in Fig. 3 show the actuation force acting on the robot for all possible positions over the working area, all steps required for the robot movement, and finally four different control sequences (see Tab. I). The detailed analysis of characteristics shows that robots can be actuated over the whole area except for freezing areas. The reduction of the force $F_{\mathrm{x}}$ is by $75 \%$ of the maximum value and, therefore, the robot can be locked there.

From the comparison of individual sequences in Fig. 3, the significant advantage of the proposed technique, the possibility to change the position of the freezing area, can be seen. In the analyzed case, five different positions of the freezing area can be chosen only by the control sequence.

\section{EXPERIMENTAL VERIFICATION}

The prototype Gryllus ${ }^{2}$ described in Sec. II-B was designed to verify the proposed technique. The prototype

\footnotetext{
${ }^{2}$ The acting force $F_{\mathrm{X}}$ represents the component that causes actuation of the robots in the $x$-direction and the attractive force represents the component that causes attraction or repulsion of the robots in the $z$-direction (this component affects the robot friction).
}
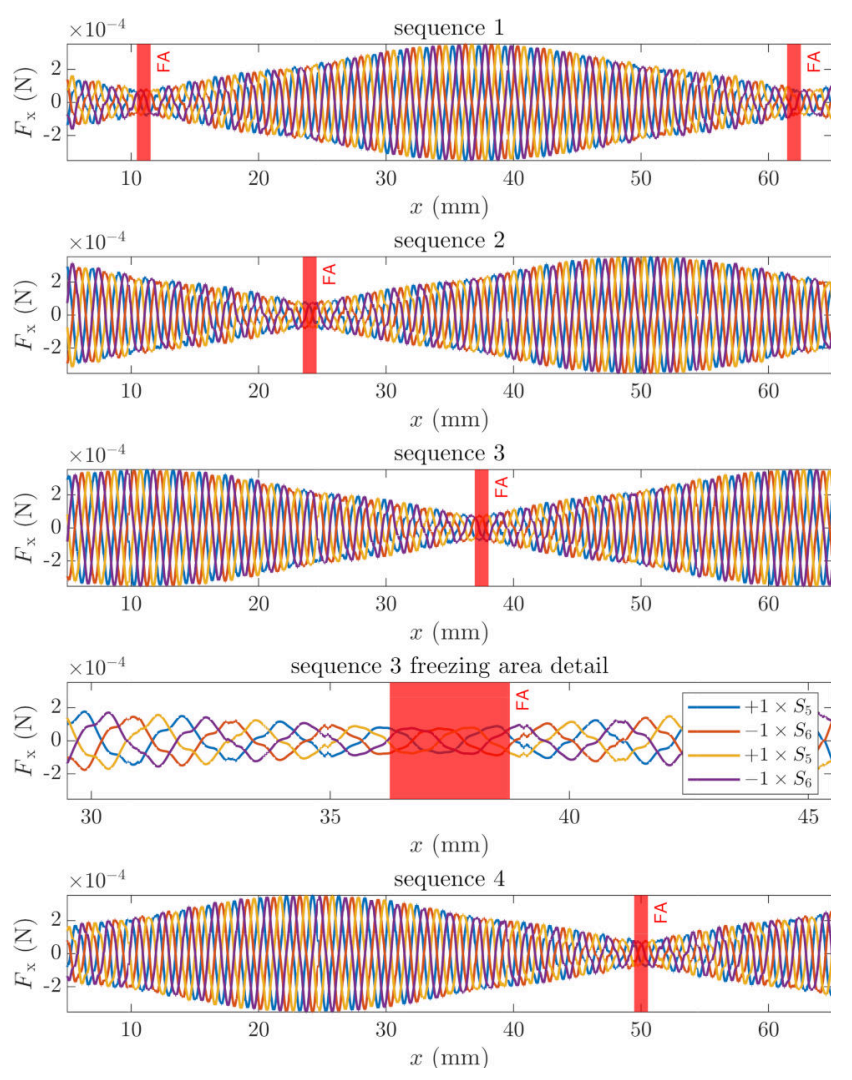

Fig. 3. Force acting on the robot calculated by numerical simulation. The red line indicates the point where the wires with opposite currents of the first and second layers intersect exactly - freezing area (FA). The freezing area can be seen in detail when the sequence 3 is used. To achieve one step, it is necessary to switch on all coils together (see tab.I).

allows 2-DOF actuation by two pairs of coplanar coils for each direction. The actuation and freezing coils differ in thread pitch, $d_{L_{1}}$, and $d_{L_{2}}$. The freezing coils in the second and fourth layer of PCB have step-index 1.04 compared to the actuation coils in the first and third layer (freezing coil has thread pitch $d_{L 2}=0.26 \mathrm{~mm}$, actuation coils $d_{L_{1}}=$ $0.25 \mathrm{~mm}$ ). The distance between freezing areas is $13 \mathrm{~mm}$ due to the selected step-index of the coils. The actuation and freezing coils for the $x$-direction are shown in Fig. 4.

The topology of the coils corresponds to the simulation presented in Sec. III-A. Five freezing areas in the $x$-direction and six areas in the $y$-direction (see Fig. 3 and Fig. 10) can be created in the working area of $75 \times 80 \mathrm{~mm}$. Each freezing area fills $4.2 \%$ of the working area (see Fig. 6).

\section{A. Magnetic field measurement}

Measurement of the magnetic flux density $\boldsymbol{B}$ along the working area was performed to verify the origin of freezing areas determined by simulations presented in Sec. III-A (the force acting on the robot is very small, for direct measurement, see Fig. 3). Figure 5 collects all measurements for control sequence 3 and corresponds with the calculated forces presented in Fig. 3.

Magnetic flux density $\boldsymbol{B}$ was measured on the perpendicular line to the active coplanar coils in the length of $70 \mathrm{~mm}$ 


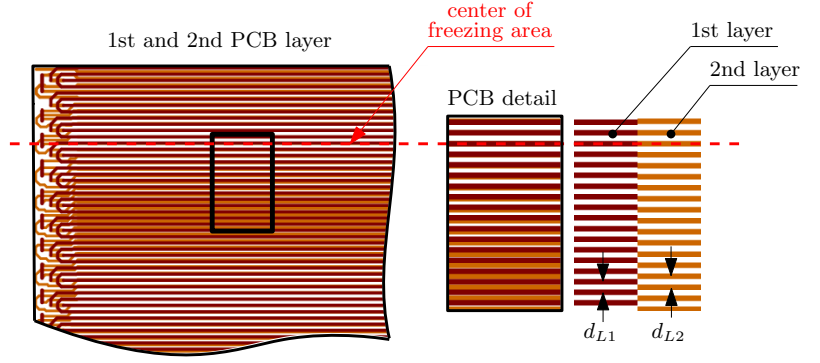

Fig. 4. Cut-out of the first and second layer of PCB and its detail. The red dashed line indicates the freezing area, which is achieved by different coil steps in the first and second layer $-d_{L 1}$ and $d_{L 2}$ respectively. Presented PCB layers correspond with topology in Fig. 2. Another orthogonal pairs of coplanar coils in two additional PCB layers are used for full 2-DOF actuation (see Sec. II-B).

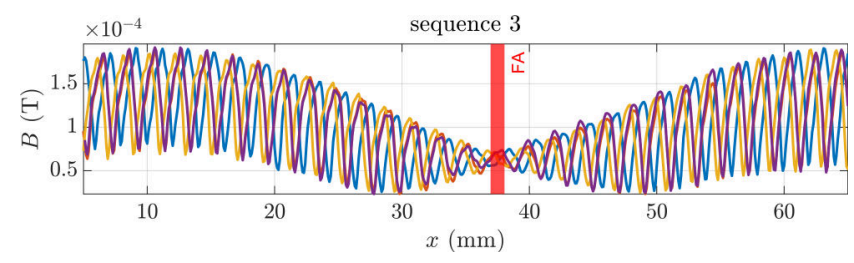

Fig. 5. Results of the magnetic flux density $B$ measurement. Freezing areas (FA) is indicating by the red line.

with spatial step $100 \mu \mathrm{m}$ and averaged data from one nine sensors in a row were used. The amplitude of measured magnetic flux density for different sequences is shown in Fig. 5. From the comparison of simulated forces (see Fig. 3) and the measurement of magnetic flux density $\boldsymbol{B}$, the effect of the freezing area is clear. It is worth mentioning that the magnetic flux density $\boldsymbol{B}$ has half frequency with respect to the acting force $F_{\mathrm{x}}$.

\section{B. Freezing area identification}

The identification of the freezing areas wide was estimated based on a simple experiment. The maximum of actuation force in each step is changed gradually between freezing areas (see Fig. 3), and it is essential to take the dynamics of the robot into account. The real width of the freezing area was then measured by repetitive movement of the robot over the whole working area, while the tested freezing areas were activated $^{3}$. The movement of the robot was captured by a camera and analyzed based on optical localization [20].

Part c) in Fig. 6 shows an example of measured data. In this measurement, the robot is moving over the working area in the $x$-direction and the freezing area defined by control sequence 3 (see part a) in Fig. 6) was periodically activated. Individual curves show the localized position of the robot stopping $x(t)$ for both directions - top-down and bottomup movement. The determined width of the freezing area is $3.2 \mathrm{~mm}$ and can be moved with the $13 \mathrm{~mm}$ steps along with the board according to the switching sequence.

\footnotetext{
${ }^{3}$ During the experiments, all freezing areas were tested individually for all directions of the robot actuation.
}

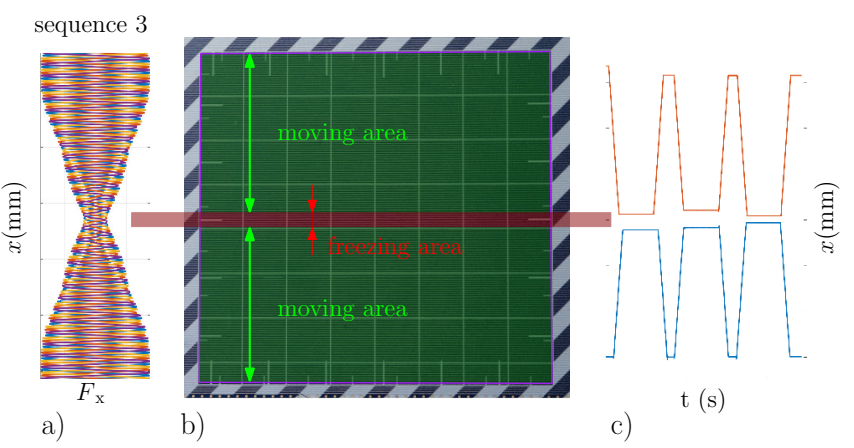

Fig. 6. The image (a) stands for the simulation of the force acting on the robot with respect to the position. In the middle (b) is the photo of the actuation board. On the right side (figure (c)) is the result of the experiment where two robots (blue and red) were actuated against each other in two consecutive experiments and always stopped at the border of the freezing area, thus the size of the freezing area was determined. All images are aligned based on the position of the freezing area.

\section{Collective operation}

The collective multi-robot operation by uniform control is illustrated first on the simple experiment in Fig. 7 and Fig. 8. Four identical robots are operated at the same time in the working area. In the initial stage, robot $\mathrm{C}$ is located in the freezing area, and others are moving simultaneously. In the second stage, the robot A entered the same freezing area and stopped even when the direction of the other robots movement is changed. In the last stage, all robots are moving (only actuation coils are excited). The experiment was performed at speeds of $25 \mathrm{~mm} \mathrm{~s}^{-1}$ with power input $7.4 \mathrm{~W}(1.4 \mathrm{~W}$ for actuation and $6 \mathrm{~W}$ for freezing coils).

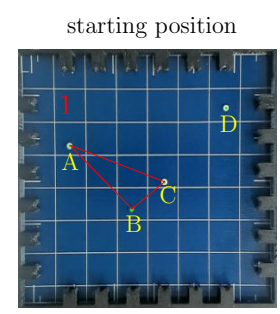

sequence 3

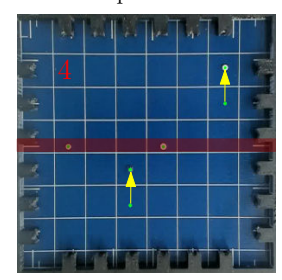

Fig. 7. An illustrative experiment of multiple robots operation by uniform control input. Each image represent a single frame from video recorded during the experiment. Red rectangle stands for freezing area, green dots represent previous and new position of all robots and yellow arrows shows their trajectories.

The experiment illustrates the formation changes of the multiple robots (see Fig. 7 first and last picture) only by the dynamic formation of the freezing area (compare the relative positions of the robot $\mathrm{A}, \mathrm{B}$, and $\mathrm{C}$ between the first and last frame in Fig. 7). These operations allow to apply algorithms designed for underactuated multi-robots systems. [16] 

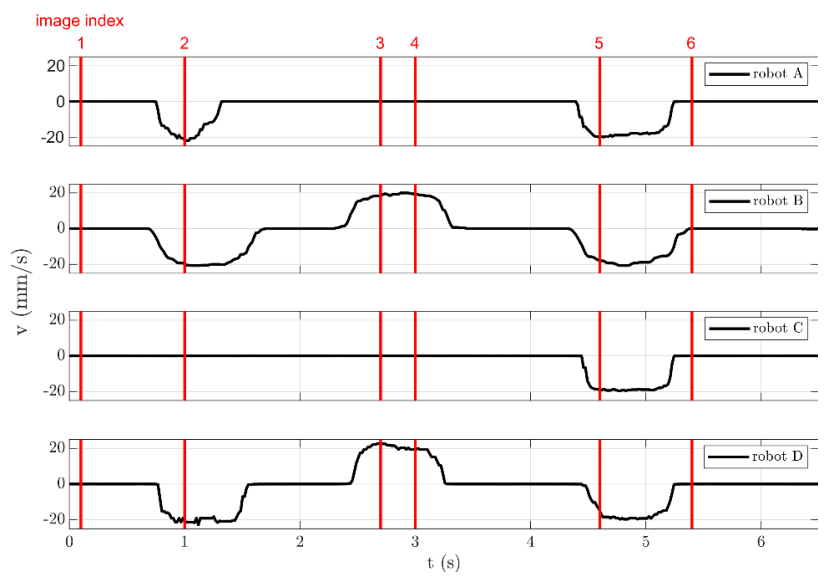

Fig. 8. Functions of speed $v$ dependent on time $t$ for the experiment presented in Fig. 7. Each subplot corresponds to the robot marked in Fig. 7. With red lines are marked moments when the images were taken.

The more complex experiment shows robot cooperation in a simple manipulation task (see Fig. 9). In this illustrative task, it is necessary to manipulate two objects - heavy and light, while the light object must be placed inside the heavy one. Two different robots are used. At the beginning, the small robot is placed inside the freezing (sequence 1 is used) and the large robot manipulates the heavy part. Subsequently, the large robot moves into the freezing area and the small robot manipulates the light object. The freezing areas are dynamically change during the experiment and the lock-up field is used to eliminate robots mutual interaction [14]. The currents were set to $0.3 \mathrm{~A}$ for 1 st PCB layer, $0.45 \mathrm{~A}$ for 2 nd layer, 0.6 A for 3rd layer, and 0.8 A for 4th layer (elimination of different $z$ position of the layers $-z$ direction).

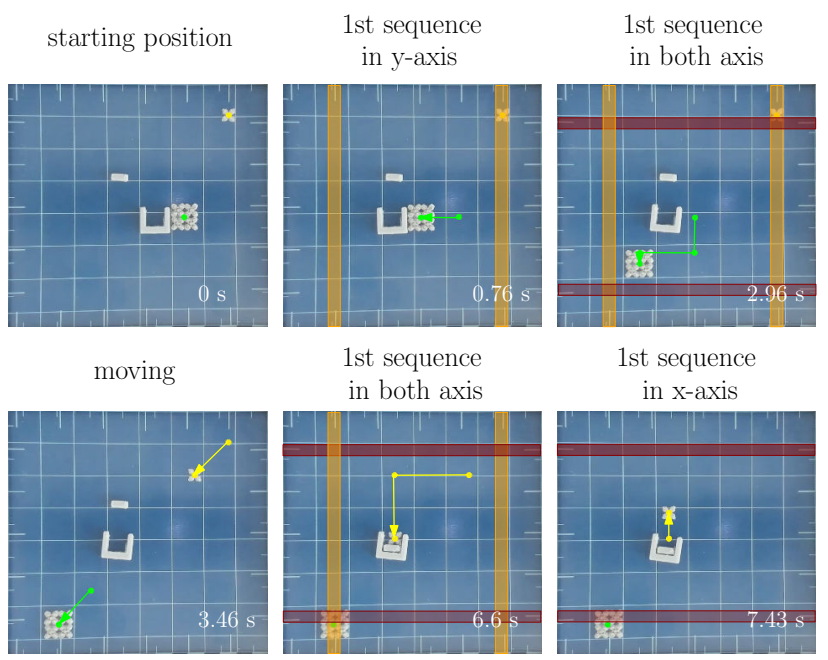

Fig. 9. An illustrative experiment of two robots cooperation in manipulation tasks. Each image represents a single frame from the video recorded during the experiment. The red rectangle stands for freezing areas in the $\mathrm{x}$-axis and orange in the y-axis.

\section{CONCLUSION}

The underactuated multi-robot system operated by the external magnetic field was discussed. The major goal was to propose the technique that dynamically disturbs the external magnetic field and thereby enables collective (but independent) operation of the multiple identical miniature robots. The technique is based on coplanar coils topology with different thread pitch. The topology was numerically analyzed (see Sec.III-A) and experimentally verified (see Sec.IV). Based on the numerical simulations, the control sequence was found (see Tab. I) and basic experiments with the collective multi-robot operations were presented. The experiment is recorded and analyzed in the attached video.

The proposed technique and the robotic system can be used for collective manipulation at multiple scales. The main advantages and potential limitations of the technique can be summarized in the following points:

- The position of the freezing area can be dynamically changed with discrete steps over the actuation board (see Fig. 3 and Fig. 9).

- Robots outside the freezing area can be actuated on more than $90 \%$ of the total working area (see Fig. 6).

- Only a small number of electronic control elements have to be used because only two coplanar coils for each degree of freedom are used. The system can be also easily reduced or expand.

- Higher input power has to be applied when the freezing is used (see Sec. IV-C).

\section{APPENDIX}

\section{A. Experimental setup and measurement methods}

All presented measurements and experiments were realized with the prototype Gryllus ${ }^{2}$. The prototype consists of a micro-controller board (ATmega328P), drivers (H-bridge L298M) and a working area designed as a multi-layer printed circuit board $(\mathrm{PCB})$ of total dimensions $100 \times 100 \mathrm{~mm}$ (each layer of the PCB contains one pair of coplanar coils). The characteristic properties of the Gryllus ${ }^{2}$ prototype are collected in Table II. Figure 10 shows prototype photography.

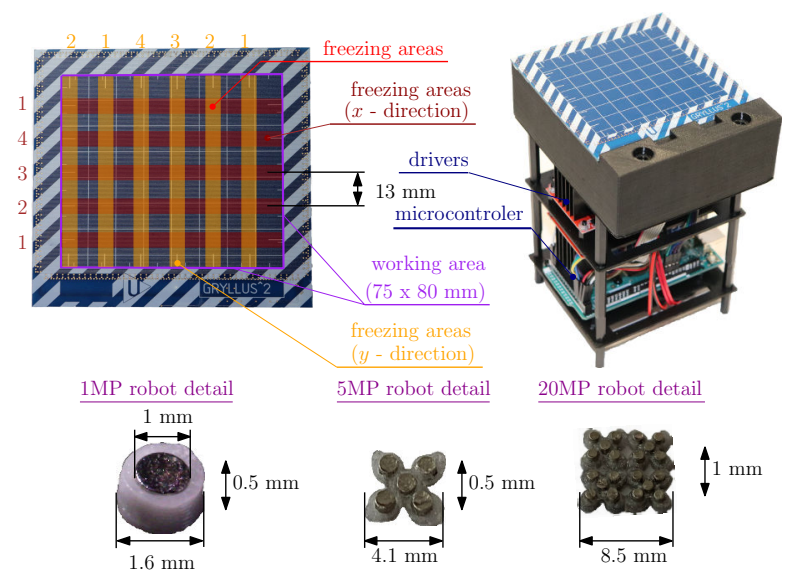

Fig. 10. Prototype Gryllus ${ }^{2}$. With highlighted freezing areas that can be switched individually and all robots that were used during the experiment.

The robots used in experiments consist of a non-magnetic body with neodymium permanent magnets (VMM7-N42) of cylindrical shape. The robot body is manufactured by 
FDM printer from polylactic acid (PLA) with dimensions $1.6 \times 1.0 \mathrm{~mm}$ (see Fig. 10).

TABLE II

PARAMETERS OF Gryllus ${ }^{2}$ PROTOTYPE.

\begin{tabular}{c|c|c}
\hline \multicolumn{3}{c}{ PCB parameters } \\
\hline Wire width & \multicolumn{2}{c}{$0.25 \mathrm{~mm}$} \\
Wire gap & \multicolumn{2}{c}{$0.25 \mathrm{~mm}$} \\
Layer gap & \multicolumn{2}{|c}{$35 \mathrm{~mm}$} \\
Copper height & \multicolumn{2}{|c}{$35 \mathrm{~m}$} \\
\hline Parameter of coils with $d_{L 1}$ & Direction $x$ & Direction $y$ \\
\hline Resistance & $17.5 \Omega$ & $47.2 \Omega$ \\
Length & $8200 \mathrm{~mm}$ & $8253 \mathrm{~mm}$ \\
\hline Parameter of coils with $d_{L 2}$ & Direction $x$ & Direction $y$ \\
\hline Resistance & $48.2 \Omega$ & $48.8 \Omega$ \\
Length & $8100 \mathrm{~mm}$ & $8145 \mathrm{~mm}$ \\
\hline \hline \multicolumn{3}{|c}{ 1PM robot parameter } \\
\hline Magnet dimensions & $1 \times 0.5 \mathrm{~mm}$ \\
Magnet weight & $3 \mathrm{mg}$ \\
Robot body weight & $0.8 \mathrm{mg}$ \\
\hline
\end{tabular}

1) Magnetic parameters measurement: Magnetic flux density $\boldsymbol{B}$ at the surface of the coplanar coils presented in Fig. 5 was measured with in-house developed scanner of magnetic field distribution in planar surfaces [19]. This scanner has array of $10 \times 10$ triple axis magnetometers and this array is actuated with $x-y$ table with a spatial resolution up to $5 \mu \mathrm{m}$. Magnetometers have dynamic range $\pm 5 \mathrm{mT}$ with resolution $150 \mathrm{nT}$.

2) Robot localization: For all the measurements was used visual localization method presented in [20]. It is a two-stage technique. First, the robot is quickly located with single pixel precision and second, the optical flow algorithm allows us to get sub-pixel precision. The result is fast $(60 \mathrm{FPS}$ at a resolution of $400 \times 400 \mathrm{px}$ ) and more precise than the pixel size. The movement of the robots in the working area was recorded by a $4 \mathrm{~K}$ camera Logitech Brio with frame rate 60 FPS.

\section{B. Mathematical model}

The distribution of the magnetic field on the surface of the coplanar coil system for any position of the robot was solved by the partial differential equation in the form

$$
\operatorname{curl}\left(\mu^{-1}\left(\operatorname{curl} \boldsymbol{A}-\boldsymbol{B}_{\mathrm{r}}\right)\right)=\boldsymbol{J}_{\text {ext }},
$$

where $\mu$ denotes the magnetic permeability, $\boldsymbol{A}$ stands for the magnetic vector potential, $\boldsymbol{B}$ and $\boldsymbol{B}_{\mathrm{r}}$ represents the magnetic flux density and remanent magnetization, respectively, and $\boldsymbol{J}_{\text {ext }}$ represents the density of the field current in the appropriate coil.

The force $\boldsymbol{F}$ acting on permanent magnets is the superposition of Lorentz $\boldsymbol{F}_{\mathrm{L}}$ and Maxwell force $\boldsymbol{F}_{\mathrm{M}}$. The Maxwell force was neglected within the modeled situations ${ }^{4}$ and acting Lorentz force is described in the form

$$
\boldsymbol{F}_{\mathrm{L}}=\boldsymbol{J} \times \boldsymbol{B} .
$$

\footnotetext{
${ }^{4}$ Robots are composed only from permanent magnets with relative permeability $\mu_{\mathrm{r}} \approx 1.2-1.4$ and then Maxwell force is very small $\left(\boldsymbol{F}_{\mathrm{M}}<0.1 \cdot \boldsymbol{F}_{\mathrm{L}}\right)$.
}

The numerical solution was performed by COMSOL Multiphysics 5.5 and MATLAB R2019b for all numerical simulations presented in the Sec. III-A. Finite element method was used for the solution of (1) and acting force described by (2) was calculated in the volume of excited coils.

\section{REFERENCES}

[1] $\mathrm{Hu}, \mathrm{V} .$, et al. Small-scale soft-bodied robot with multimodal locomotion.. Nature, 554.7690, 2018.

[2] Dong, X., Sitti, M., Controlling two-dimensional collective formation and cooperative behavior of magnetic microrobot swarms, The International Journal of Robotics Research, vol. 3, no. 2020.

[3] K. Han, et al., Sequence-encoded colloidal origami and microbot assemblies from patchy magnetic cubes, Science advances, vol. 3, no. 8, 2017.

[4] S. Tasoglu, et al. Untethered micro-robotic coding of three-dimensional material composition, Nature communications, vol. 5, 2014.

[5] Pelrine. R. Super materials and robots making robots: Challenges and opportunities in robotic building at the microstructural level, Robotic Systems and Autonomous Platforms, 2019.

[6] R. Pelrine, et al., Multi-agent systems using diamagnetic micro manipulation-From floating swarms to mobile sensors, International Conference on Manipulation, Automation and Robotics at Small Scales (MARSS), 2017.

[7] Yu, J., et al., Ultra-extensible ribbon-like magnetic microswarm, Nature communications, vol. 9, no. 1, 2018.

[8] Li, J., et al., Micro/nanorobots for biomedicine: Delivery, surgery, sensing, and detoxification, Science Robotics, vol. 2, no. 4, 2017.

[9] Yang, G. Z., et al. The grand challenges of Science Robotics. Science Robotics, 3.12, 2018.

[10] J. Rahmer, C. Stehning, B. Gleich, Spatially selective remote magnetic actuation of identical helical micromachines, Science Robotics, vol. 2, no. 3, 2017.

[11] A. Steager, et al., Control of multiple microrobots with multiscale magnetic field superposition, International Conference on Manipulation, Automation and Robotics at Small Scales (MARSS), 2017.

[12] Kuthan, J., et al. Magnetic Actuation of Multiple Robots by the Coplanar Coils System., International Conference on Manipulation, Automation and Robotics at Small Scales (MARSS), 2019

[13] R. Pelrine, et al., 'Diamagnetically levitated robots: An approach to massively parallel robotic systems with unusual motion properties', IEEE International Conference on Robotics and Automation (ICRA), 2012.

[14] M. Juřik, J. Kuthan, J. Vlček and F. Mach., 'Positioning Uncertainty Reduction of Magnetically Guided Actuation on Planar Surfaces', IEEE International Conference on Robotics and Automation (ICRA), 2019.

[15] A. V. Mahadev, A. Mahadev, et al., Collecting a swarm in a grid environment using shared, global inputs. IEEE International Conference on Automation Science and Engineering (CASE), pp. 1231-1236, 2016.

[16] A. Becker, et al., Massive uniform manipulation: Controlling large populations of simple robots with a common input signal, IEEE/RSJ International Conference on Intelligent Robots and Systems (IROS), 2013.

[17] S. Chowdhury, W. Jing, D. J. Capperlleri, Towards independent control of multiple magnetic mobile microrobots, Micromachines, vol. 7, no. $1,2015$.

[18] S. Chowdhury, et al., Designing local magnetic fields and path planning for independent actuation of multiple mobile microrobots, Journal of Micro-Bio Robotics, vol. 12, 2017.

[19] Vítek, M., et al. Precise Scanner of Magnetic Field Distribution., International Conference on Manipulation, Automation and Robotics at Small Scales (MARSS), 2019

[20] Juř́ík, M., et al. Trade-off Between Resolution and Frame Rate for Visual Tracking of Mini-robots on Planar Surfaces., International Conference on Manipulation, Automation and Robotics at Small Scales (MARSS), 2019 